\title{
Lichen plan buccal : histoire naturelle et transformation maligne
}

\section{Oral lichen planus: natural history and malignant transformation}

\author{
STELLA LYSITSA, SEEMAN ABI NAJM, TOMMASO LOMBARDI, JACKY SAMSON
}

\section{RÉSUMÉ}

Le lichen plan est une dermatose cutanéo-muqueuse inflammatoire chronique qui touche toutes les régions de la muqueuse buccale. II y a quatre phases dans l'évolution du lichen plan buccal (LPB) : la phase initiale, la phase d'état, la phase tardive et l'état post-lichénien.

Plusieurs hypothèses ont été proposées pour tenter d'expliquer l'étiopathogénie de LPB et son association à d'autres maladies. En conséquence, il n'y pas de traitement bien codifié.

Quelques études entretiennent la controverse et créent une confusion sur le potentiel de transformation maligne du LPB. Comme le LPB est une affection fréquente, souvent banalisée par le chirurgien dentiste, il semblait opportun d'effectuer une revue de la littérature en s'intéressant à son histoire naturelle, ses caractéristiques cliniques et histologiques, son étiopathogénie, son association à d'autres maladies, son traitement et sa transformation maligne. Med Buccale Chir Buccale 2007; 13: 19-29).

médecine buccale chirurgie buccale

VOL. $13, \mathrm{~N}^{\circ} 1$ 2007 page 19

mots clés: lichen plan buccal, état post-lichénien, lésions lichénoïdes, transformation maligne

\section{SUMMARY}

Oral lichen planus $(O L P)$ is a chronic inflammatory dermato-mucosal disease which gets all the regions of the buccal mucous membrane. There are four phases of the evolution of the OLP: the initial phase, the phase of state, the late phase and the post-lichenien state. In the literature there are several hypotheses on the etiopathogeny of OLP as well as on the association in the other diseases. There are several treatments proposed.

Controversial studies create confusion about the malignant transformation of the OLP. Given that the OLP represents a frequent disease, with whom the dentist often confronts, our work concerns a detailed review of the literature on the natural history of the OLP, the clinical and histological aspect, its etiopathogeny, its association with the other diseases, the treatment and finally its malignant transformation. Med Buccale Chir Buccale 2007; 13: 19-29).

key words: oral lichen planus, post-lichen state, lichenoid lesions, malignant transformation 


\section{DONNEES EPIDEMIOLOGIQUES}

Le lichen plan (LP) est une dermatose cutanéomuqueuse inflammatoire chronique, dont la description clinique initiale a été faite par Wilson en 1869 et la description histologique par Dubreuil en 1906. Son incidence varie, selon les auteurs, de $0,1 \%$ à $4 \%$, avec une prédilection pour les sujets d'âge moyen (quatrième et cinquième décennies) et les femmes [45]. L'étude démographique réalisée par Axell et Rundquist donne une prévalence de $1 \%$ à $2 \%$ sur une période de 15 ans ${ }^{[4]}$. La moitié des sujets ayant des lésions cutanées développe également des lésions buccales, mais $25 \%$ des sujets présentent uniquement des lésions buccales [3]. Contrairement aux lésions cutanées qui guérissent le plus souvent spontanément en 1 à 2 ans, les lésions muqueuses ont une évolution prolongée sur plusieurs années [1]. On retrouve dans la médecine

buccale

chirurgie buccale

VOL. $13, \mathrm{~N}^{\circ} 1$ 2007

page 20 littérature la description d'un syndrome vulvovagino-gingival qui correspond en réalité à une forme particulière de LPB ${ }^{[43]}$. Le LP peut avoir différentes localisations et environ $6 \%$ des patients ayant un LPB présentent des lésions lichéniennes sur au moins trois autres sites (atteintes cutanée, œsophagienne, génitale, ophtalmique). Ceci confirme la nécessité d'une approche multidisciplinaire pour cette maladie ${ }^{[12] .}$

\section{HISTOIRE NATURELLE}

Le LPB peut toucher toutes les régions de la muqueuse buccale, y compris la demi-muqueuse labiale. Les formes localisées sont plus fréquentes que les formes diffuses, et la région jugale postéro-inférieure représente le siège électif, avec souvent une atteinte bilatérale et grossièrement symétrique. Par ordre décroissant de fréquence, les localisations sont les suivantes: muqueuse jugale postéro-inférieure, dos de la langue, fibromuqueuse gingivale, fibromuqueuse palatine, face ventrale de la langue, muqueuse ou demi- muqueuse labiale, et plancher buccal. L'histoire naturelle du LPB comporte trois phases : la phase initiale, la phase d'état avec ses périodes de quiescence et d'activité, et la phase tardive [11].

\section{Aspects cliniques}

La phase initiale qui dure 6 à 12 mois, se traduit par des lésions blanches, punctiformes, hémisphériques, siégeant le plus souvent dans la région jugale postérieure ; sur le dos de la langue, les lésions kératosiques intéressent principalement l'extrémité des papilles filiformes (lichen papillaire de Gougerot). Ces lésions vont progressivement s'étendre et confluer pour former des lignes (stries de Wickham), puis dessiner différentes figures (LPB réticulé, dendritique, circiné...), ou former des plages kératosiques. Sur le dos de la langue, la kératose envahit toute la surface des papilles et les espaces interpapillaires, puis très rapidement il apparaît une dépapillation irréversible sur les plages kératosiques initiales ; la kératose persiste et peut prendre parfois l'aspect de taches en cire de bougie ou en pain à cacheter. En dehors des plages kératosiques souvent discrètes, l'atteinte de la fibromuqueuse gingivale se traduit par la disparition de son aspect granité et un discret changement de couleur, la fibromuqueuse devient un peu plus rouge. Toutes ces lésions ressemblent à celles de la phase d'état qui dure une dizaine d'années ou plus, avec une succession de poussées d'activité et de périodes de quiescence. Leur aspect varie dans le temps et ces lésions peuvent régresser ou disparaître momentanément sous traitement, plus rarement de façon spontanée. Chaque poussée d'activité est marquée par l'apparition de plages érythémateuses, voire d'érosions (LPB érosif), ou seulement par l'extension des lésions kératosiques préexistantes. Exceptionnellement, le plus souvent dans la région jugale postérieure, des bulles peuvent apparaître (LPB bulleux). Les signes fonctionnels sont très variables : perception d'un relief ou d'une perte de la souplesse avec la langue, gène, douleur ou sensation de brûlures dépendent de l'importance de la poussée. En dehors des poussées, il n'y a pas de symptômes et le LPB se traduit alors par des lésions kératosiques qui régressent le plus souvent, sans disparaître totalement. Quelquefois, chez les sujets ayant une peau mate ou noire, une pigmentation brunâtre ou noirâtre, mal limitée, apparaît progressivement (LPB nigricans) ; son développement est favorisé par la fréquence et 
l'intensité élevées des poussées inflammatoires. La phase tardive commence après plusieurs années d'évolution, et quelquefois sans que le LPB n'ait été diagnostiqué. Elle se traduit par le développement d'un état atrophique ou scléroatrophique. L'atrophie intéresse principalement les zones de la muqueuse buccale où il y a eu des lésions actives, donc le plus souvent la face interne des joues. La couleur de la muqueuse change et perd son homogénéité : on observe des plages de couleur discrètement jaunâtre, brunâtre ou rougeâtre; l'atrophie permet parfois de voir par transparence le réseau vasculaire sousmuqueux. Sur le dos de la langue, l'atrophie se traduit par des plages dépapillées, recouvertes ou non par une couche kératosique parfois épaisse. L'atrophie de la fibromuqueuse gingivale entraîne une rétraction gingivale souvent associée à une diminution de la profondeur des vestibules, surtout visible dans les régions postéro-inferieures. L'atrophie favorise le développement d'érosions pour des traumatismes mineurs. La sclérose est plus ou moins marquée ; au maximum elle se traduit par une perte de l'élasticité avec limitation de l'ouverture buccale (kraurosis oris) et une diminution de la protraction linguale. Lors du passage au stade post-lichénien, et surtout pendant le stade post-lichénien, la muqueuse scléro-atrophique peut être le siège de foyers de transformation maligne aboutissant au développement d'un carcinome verruqueux ou d'un carcinome épidermoïde, souvent bien différencié ${ }^{[44]}$.

\section{Aspects histopathologiques}

Les altérations intéressent surtout l'épithélium. A la phase initiale, on observe les éléments suivants : parakératose de surface, épaississement ou amincissement, effilochage des crêtes interpapillaires, présence d'une couche granuleuse irrégulière qui, avec l'infiltrat inflammatoire sousépithélial nodulaire, sont responsables de l'apparition des lésions blanches, punctiformes ou réticulées, caractéristiques de la phase initiale. $A$ la phase d'état, il existe une ortho- ou une parakératose de surface, les crêtes prennent progressivement un aspect en dents de scie, les papilles une forme en dôme et on observe dans
I'assise basale quelques corps hyalins qui correspondent à des kératinocytes en apoptose ; le chorion superficiel est le siège d'un infiltrat inflammatoire en bande, à prédominance lymphocytaire. Les poussées d'activité sont marquées par une diminution de la kératose de surface, la présence éventuelle d'érosions, d'une spongiose, d'une exocytose, d'une augmentation du nombre de corps hyalins et d'une augmentation de la densité de l'infiltrat inflammatoire. Progressivement, l'aspect initial (papilles en dôme et crêtes interpapillaires en dents de scie) évolue et la membrane basale tend à devenir rectiligne. A la fin de l'évolution, l'épithélium est aminci, la membrane basale rectiligne, la kératose parfois verruqueuse, le chorion le siège d'une fibrose collagène plus ou moins importante tandis que l'infiltrat inflammatoire s'éclaircit. Au stade post-lichénien, la muqueuse est atrophique et l'infiltrat inflammatoire a disparu [33]. Ce stade est caractérisé par une atrophie épithéliale, une hyperkératose de surface et une fibrose de chorion [28].

\section{Etiopathogénie}

L'étiopathogénie de LP n'est pas totalement élucidée. Deux hypothèses ont été proposées pour expliquer le développement du LP : classiquement, on considérait qu'il pourrait résulter d'une altération des kératinocytes, d'origine inconnue, avec libération ou formation d'antigènes entraînant une réponse immunitaire, ou d'une réaction immunologique primaire responsable de l'altération des kératinocytes et de leur apoptose [6]. Cette réponse immunitaire, à médiation cellulaire de type $T$, a orienté la recherche sur le rôle des lymphocytes dans le développement du LP. D'après les experts qui ont participé à la réunion de consensus qui a eu lieu en 2005 sur l'étiopathogénie du LPB, les cellules présentant les antigènes et les kératinocytes de la couche basale pourraient être activées par une infection, des molécules d'origine bactérienne, des traumatismes mécaniques, un traitement systémique, une sensibilité de contact ou des agents non identifiés. Ces cellules activées produiraient des chémokines qui attireraient et stimuleraient les lymphocytes. L'infiltrat est constitué principalement de lymphocytes cytotoxiques CD8 et de lymphocytes médecine

buccale chirurgie buccale

VOL. $13, \mathrm{~N}^{\circ} 1$ 2007

page 21 
médecine

buccale

chirurgie buccale

VOL. $13, N^{\circ} 1$ 2007

page 22 non cytotoxiques CD4. Les cellules de Langerhans et les kératinocytes du LPB présentent un antigène associé au complexe majeur d'histocompatibilité $(\mathrm{CMH})$ de type II et aux lymphocytes $\mathrm{T}$ CD4+ qui sont stimulés et produisent de l'interleukine 2 (IL-2) et de l'interféron (IFN) gamma. Les lymphocytes T CD8+ pourraient être activés par un antigène associé au $\mathrm{CMH}$ de type I des kératinocytes de la couche basale ou par l'IL-2 et l'INF gamma [30]. Les lymphocytes T CD8+ libèreraient du TNF alpha qui provoquerait l'apoptose des kératinocytes [37]. L'antigène présenté par les $\mathrm{CMH}$ de types I et II pourrait être un peptide, ce qui confirmerait la nature autoimmune de LP. Plusieurs éléments sont compatibles avec cette hypothèse : la chronicité de la maladie, l'âge et le sexe de prédilection, l'association éventuelle à d'autres maladies autoimmunes, la présence des cellules $T$ autotoxique [51].

\section{LPB « induit » ou associé à d'autres maladies}

Souvent, des lésions identiques cliniquement et histologiquement aux lésions du LPB peuvent se développer sur la muqueuse buccale. Ces lésions, dites lichénoïdes, sont généralement assimilées au LPB - qui est alors qualifié de LPB «induit »-, ce qui crée une confusion regrettable pour la compréhension et le traitement du LPB. Les lésions lichénoïdes sont moins fréquentes sur la muqueuse buccale que sur la peau. Elles peuvent être induites soit par des traitements médicamenteux systémiques, soit par des matériaux utilisées pour les restaurations dentaires. Les médicaments les plus fréquemment en cause sont les antipaludéens de synthèse, les antidépresseurs, les médicaments prescrits pour la polyarthrite rhumatoïde (sels d'or, D-pénicillamine, INF), certains antituberculeux, des antihypertenseurs, des diurétiques, des médicaments hypoglycémiants, des anti-inflammatoires non stéroïdiens, l'allopurinol... [34]. Dans les matériaux utilisés pour les restaurations dentaires, les plus cités sont le mercure, l'or, le chrome et le sulfate de cuivre. Les lésions induites par ces matériaux se présentent sous la forme de lésions blanches ou érythémateuses, parfois associées à des érosions ou à des ulcérations. Elles sont limitées à la zone de contact avec le matériau de restauration et associées à des douleurs ou à une sensation de sécheresse buccale. Elles sont souvent liées à une irritation chronique ou une réaction d'hypersensibilité retardée (type allergie de contact) ; dans ce cas, on peut réaliser un patch test, mais cet examen n'est pas très fiable [20]. L'élimination du matériau responsable aboutit le plus souvent à la guérison des lésions ${ }^{[7,40]}$.

Le LPB peut être associé à une maladie systémique. Le syndrome de Grinspan qui comporte un LPB, un diabète et une hypertension artérielle, constitue la première association décrite. Ce syndrome semble en réalité d'origine iatrogène : ce serait les médicaments prescrits pour le traitement du diabète ou de l'hypertension qui induiraient les lésions lichénoïdes [29]. De nombreuses études ont été publiées sur l'association LPB et hépatite $C$, mais la controverse persiste sur l'existence de cette association. Elle est retrouvée dans les pays de l'Europe méditerranéenne [35] et au Japon [38] (ou respectivement $34 \%$ et $62 \%$ des patients atteints d'une hépatite $\mathrm{C}$ développent un LPB) mais elle n'est pas observée dans d'autres pays comme par exemple l'Angleterre [20]. Cette différence de fréquence liée à l'origine géographique pourrait dépendre de facteurs immunogénétiques.

L'association LPB et HPV (human papilloma virus) a été aussi signalée. Même si certaines études montrent une fréquence importante de HPV (surtout HPV 16 et 18) dans le LPB, l'existence de cette association n'est pas réellement documentée. La présence des HPV pourrait être fortuite ou favorisée par le traitement immunosuppresseur du LPB [30]. Certains auteurs proposent une surveillance attentive pour les patients qui sont HPVpositifs en raison du potentiel oncogénique de certains HPV [10].

Dans la GVHD (graft-versus-host disease), on observe souvent des lésions buccales qui ressemblent cliniquement et histologiquement au LPB [39]. Elles sont retrouvées dans $33 \%$ à $75 \%$ des cas pendant la phase aiguë (100 premiers jours après la transplantation) et chez $85 \%$ des patients pendant la phase chronique de la GVHD (après les 100 premiers jours suivant la trans-

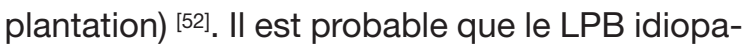


thique et la GVHD partagent les mêmes mécanismes immunopathologiques : infiltrat inflammatoire composé de cellules $\mathrm{T}$, apoptose des kératinocytes et altération de la membrane basale. La GVHD buccale présente également un risque de transformation maligne. Mais dans ce cas, il y a d'autres facteurs (immunodéficience primaire, traitement immunosuppresseur, infections virales, prédisposition génétique au cancer), susceptibles de favoriser la transformation maligne. Néanmoins, il existe une grande similitude entre ces deux affections - aspects cliniques et histopathologiques, potentiel de transformation maligne - qui doit être prise en considération [31] et les données obtenues sur l'une d'elle permettent d'illustrer des éléments physiopathologiques de l'autre [53].

Enfin, plusieurs études s'intéressant à la relation entre le LPB et l'état psychique montrent que le stress et la dépression représentent des facteurs favorisant le développement du LPB ${ }^{[55] .}$

\section{TRAITEMENT ET SUIVI DES SUJETS ATTEINTS D'UN LPB}

II n'y a pas de traitement curatif pour le LPB. Un traitement est préconisé lorsqu'il existe une symptomatologie ou lorsque l'on souhaite faire régresser les lésions. Le traitement de LPB est en général plus difficile que celui du LP cutané. La découverte d'un LPB ou d'une réaction lichénoïde, confirmé histologiquement, doit faire rechercher une étiologie médicamenteuse mais l'arrêt du traitement inducteur n'entraîne pas toujours une évolution favorable. Elle doit également faire réaliser une sérologie pour l'hépatite $C$, même dans les pays où cette relation n'apparaît pas évidente car elle permet parfois de découvrir une hépatite $\mathrm{C}$ latente, donc de la traiter, et de prescrire une corticothérapie générale sans risque ${ }^{[44]}$. Avant de procéder au traitement, il faut éliminer ou diminuer les facteurs locaux favorisants : la mauvaise hygiène buccodentaire, les prothèses dentaires mal adaptées et les traumatismes de la muqueuse buccale liés au bruxisme, à un tic de succion-aspiration ou à un morsicatio linguae ou buccarum qui, en irritant la muqueuse, favorisent le développement de lésions ou d'une poussée d'activité (phénomène isomorphe de Koebner). Pour les lésions symptomatiques, un traitement médicamenteux local ou systémique doit être prescrit. La corticothérapie constitue le traitement de choix. Les corticoïdes locaux donnent des bons résultats. Ils existent sous la forme de crème, de gel, de bain de bouche ou d'injections sous-lésionnelles mais ils favorisent - excepté les injections sous-lésionnelles - l'apparition d'une candidose buccale. Pour les lésions gingivales qui résistent souvent au traitement topique et systémique, on peut utiliser une gouttière pour augmenter le temps de contact entre les lésions et les corticoïdes [14]. En présence d'érosions importantes ou lorsqu'il existe d'autres lésions cutanéo-muqueuses associées, une corticothérapie systémique est indiquée. D'autres traitements comme la griséofulvine, le lévamisole, la dapsone, l'azathioprine, la ciclosporine, les rétinoïdes, la thalidomide... ont été proposés. Dernièrement, l'intérêt s'est focalisé sur un macrolide immunosuppresseur, le tacrolimus [22]. L'efficacité de cette molécule est due à son implication dans l'inhibition de l'activation des lymphocytes T. Kaliakatsou et al. [22] ont réalisé une étude sur 17 patients traités avec du tacrolimus en application topique, pendant une période de 8 semaines. Ils constatent une diminution des symptômes, sans apparition d'effets secondaires importants. Mais une réactivation de LPB est apparue chez $76,5 \%$ des patients 4 semaines environ après l'arrêt du traitement. Ce traitement doit faire l'objet d'études complémentaires pour évaluer son efficacité à long terme.

Etant donné le caractère immunosuppresseur du traitement du LPB et l'association de ce dernier au Candida, un traitement antifongique est bien souvent indiqué en première intention $[17,46]$.

Un contrôle régulier tous les 6 à 12 mois est recommandé pour suivre l'évolution du LPB au stade atrophique ou de l'état post-lichénien afin de dépister précocement tout foyer de transformation maligne et de le traiter au stade d'OIN (oral intraepithelial neoplasia), de carcinome verruqueux ou de carcinome épidermoïde micro-invasif [27]. médecine

buccale chirurgie buccale

VOL. $13, \mathrm{~N}^{\circ} 1$ 2007

page 23 


\section{TRANSFORMATION MALIGNE DU LPB}

La transformation maligne du LPB reste un sujet de controverses malgré les nombreuses études qui lui ont été consacrées. En 1910, Hallopeau a rapporté le premier cas de carcinome développé au voisinage d'un lichen plan buccal [16]. La première revue de la littérature, bien structurée, sur la transformation maligne du LPB a été faite en 1978 par Krutchkoff et coll. [26]. Après analyse des 223 cas publiés, seulement 16 cas ont été considérés comme suffisamment documentés pour démontrer la relation existant entre le LPB et le carcinome épidermoïde. Depuis cette date, d'autres cas ont été publiés, y compris des transformations malignes survenant sur un LP génital [15], œsophagien [9] ou cutané [32]. En 1985, Silverman et coll. ont publié une étude portant sur 570 patients avec un LPB, suivi pendant une médecine buccale chirurgie buccale

VOL. $13, \mathrm{~N}^{\circ} 1$ 2007

page 24 période de 5,6 ans en moyenne : 7 patients ont développé un carcinome épidermoïde, ce qui représente un taux de transformation maligne de $1,2 \%{ }^{[48]}$. En 1988, Holmstrup et coll. une étude portant sur 611 patients, suivis pendant une période de 7,5 ans : 9 patients ont développé un carcinome épidermoïde (taux de transformation maligne de 1,5 \%) [19]. En 1991, Silverman et coll. une autre étude portant sur 214 patients, suivis pendant une période de 7,5 ans : 4 femmes et 1 homme ont développé un carcinome épidermoïde et seulement un de ces patients fumait [49]. La même année, Sigurgeirsson et Lindelöf une étude portant sur 2071 patients ayant un LP cutané suivis pendant de 9,9 ans en moyenne: ils concluent que le LP cutané présente un risque de transformation maligne extrêmement faible ; pour le LPB, ce risque serait de 0,4 \% [47]. En 1992 Voûte et coll. ont suivi 113 patients pendant une période de 7,8 ans en moyenne et ils ont observé un taux de transformation maligne de 2,6\% [58]. En 1993, Barnard et al. ont présenté 241 patients avec un LPB suivis pendant 10 ans en moyenne, 8 ont développé un carcinome épidermoïde et 1 un carcinome in situ ${ }^{[5]}$. En 1999, Hietanen et coll. rapportent 8 cas de carcinome épidermoïde développés sur un LPB pendant une période de 10 ans. Le délai moyen entre le diagnostic initial de LPB et le diagnostic du carcinome est de
3,4 ans. Ils ont comparé histologiquement ces patients avec un groupe contrôle comportant 8 patients ayant un LPB sans transformation maligne, suivis pendant la même période. Cette étude comparative n'a pas apporté d'éléments pouvant justifier ou expliquer la différence d'évolution entre les deux groupes [18]. En 2001, Mignona et coll. ont suivi 502 patients avec un LPB pendant une période de 5 ans : 24 patients ont développé un carcinome épidermoïde, ce qui correspond à un taux de transformation maligne de 4,9\% [36]. En 2003, van der Meij et coll. ont rapporté une étude sur 173 patients avec un LPB : 3 ont développé un carcinome épidermoïde après un délai moyen de 31,9 mois ce qui correspond à un taux de transformation maligne de 1,7 \% [57]. Une étude récente de Bornstein et coll. présentaient un groupe de 141 patients avec un LPB, suivis pendant une période 3,7 ans en moyenne: une transformation maligne a été observée chez 4 patients ce qui correspond à un taux de transformation maligne de 2,84\%. Sur ces 4 patients, trois présentaient une dysplasie sur la biopsie initiale. Ainsi, si on exclut ces 3 patients, le taux de transformation maligne sur 138 patients est de $0,71 \%$. Ils ont ensuite comparé ce taux avec le taux de survenue du carcinome épidermoïde dans la cavité buccale dans l'ensemble de la population suisse qui est $0,0123 \%$ sur une année, soit un taux de 0,0455\% sur une période de 3,7 ans. II existe donc bien un risque de transformation maligne lié au LPB de cette étude ${ }^{[8]}$. Les principales données fournies par ces différentes études sont présentées dans le Tab. 1.

D'autres études bien documentées confirment le potentiel de transformation maligne du LPB [23,50]. Le taux de transformation maligne de LPB varie de façon importante selon les études. En 1997, l'OMS a classé le LPB dans les états précancéreux (precancerous conditions) [41]. La transformation maligne survient, le plus souvent, sur des lésions lichéniennes atrophiques et/ou érosives ${ }^{[5]}$ ou sur un EPL ; les variations du taux de transformation maligne sont probablement, au moins en partie, liées aux différents types de LPB inclus dans ces études ; aucune d'entre elles ne précise le stade évolutif ou simplement la durée d'évolution du LPB. De même, il n'est pas précisé si les 
Tableau 1 : Principales données sur le potentiel de transformation maligne du LPB retrouvées dans la littérature Malignant transformation in oral lichen planus: main data on the malignant transformation potential found in some publications

\begin{tabular}{|c|c|c|c|}
\hline $\begin{array}{c}\text { Auteurs et année } \\
\text { de publication }\end{array}$ & $\begin{array}{c}\text { Durée moyenne } \\
\text { de suivi }\end{array}$ & $\begin{array}{c}\text { Nombre de } \\
\text { patients }\end{array}$ & $\begin{array}{c}\text { Taux de } \\
\text { transformation maligne }\end{array}$ \\
\hline Silverman 1985 & 5,6 ans & 570 & $1,2 \%$ \\
\hline Holmstrup 1988 & 7,5 ans & 611 & $1,5 \%$ \\
\hline Silverman 1991 & 7,5 ans & 214 & $2,3 \%$ \\
\hline Voûte 1992 & 7,8 ans & 113 & $2,6 \%$ \\
\hline Barnard 1993 & 10 ans & 241 & $3,3 \%$ \\
\hline Mignona 2001 & 5 ans & 502 & $4,9 \%$ \\
\hline Van der Meij 2003 & 31,8 mois & 173 & $1,7 \%$ \\
\hline Bornstein 2006 & 3,7 ans & 138 & $0,71 \%$ \\
\hline
\end{tabular}

lésions érosives sont en rapport avec une poussée d'activité pendant la phase d'état ou avec l'atrophie de la muqueuse caractéristique de l'EPL. Pour expliquer la transformation maligne, plusieurs hypothèses ont été proposées mais c'est l'inflammation chronique qui semble être le facteur essentiel pour le développement du cancer. Il existe plusieurs exemples : maladie inflammatoire intestinale chronique-cancer de côlon, gastrite atrophique-cancer gastrique, reflux œsophagien-carcinome épidermoïde œsophagien, épidermolyse bulleuse dystrophique-carcinome épidermoïde cutané... Les cellules inflammatoires et leurs cytokines entraîneraient des altérations cellulaires et stimuleraient la synthèse de l'EGF (epidermal growth factor) [37]. Des études récentes ont mis en évidence, dans les lésions de LPB, une diminution de l'expression de hMSH2, gène appartenant au système hMMR (human DNA mismatch repair), qui est assimilé à un gène suppresseur de tumeur [42]. Ce gène joue un rôle important dans la diminution des mutations et la stabilité génétique. Dans le LPB, on observe une baisse de la protéine synthétisée par ce gène, qui pourrait favoriser l'augmentation des mutations de l'ADN dans les cellules épithéliales, donc leur transformation maligne. Dans le LPB, la réponse à l'inflammation chronique et le processus de la cicatrisation pourraient augmenter le nombre de mutations des gènes. Cette hypothèse semble confirmée par des études récentes qui illustrent la relation entre les médiateurs chimiques des cel- lules T de l'inflammation et le processus de la cancérogenèse. Ces études montrent que le MIF (macrophage migration inhibitory factor), libéré par les cellules $T$ et les macrophages, diminue la transcription de la p53, protéine considérée comme la gardienne du génome [53]. Le gène p53 est considéré comme un gène suppresseur de tumeur car la p53 joue un rôle important dans la carcinogenèse en éliminant les cellules mutantes par apoptose. Des mutants de la p53 ont été retrouvés dans plus que $60 \%$ des cancers ${ }^{[59]}$. Le blocage de la p53 par le MIF (ou d'autres médiateurs) augmente le risque de développement d'un cancer sur un LPB. Valente et coll. ont étudié l'expression de la p53 dans le LPB. Ils ont constitué trois groupes de patients qui ont été biopsiés à plusieurs reprises. Dans le premier groupe, les patients n'ont pas présenté d'altérations dysplasiques pendant la période de contrôle, dans le deuxième groupe, ils ont présenté simultanément un LPB et un carcinome épidermoïde et dans le troisième groupe ils ont développé un carcinome épidermoïde plusieurs années après le diagnostic de LPB. Les auteurs ont mis en évidence une surexpression de la p53 dans le deuxième et le troisième groupe. Ces résultats confirment le rôle de la p53 dans la transformation maligne du LPB et montre que l'étude de l'expression de la p53 fournit probablement des renseignements sur l'évolution et le pronostic du LPB [54]. Acay et coll. ont réalisé une étude comparable mais en utilisant un anticorps qui détecte uniquement la forme médecine

buccale chirurgie buccale

VOL. $13, \mathrm{~N}^{\circ} 1$ 2007 page 25 
médecine

buccale

chirurgie

buccale

VOL. $13, N^{\circ} 1$ 2007

page 26 mutante de la p53 : la p53 est augmentée, mais elle est beaucoup plus élevée que dans les réactions lichénoïdes [2].

Le tabac et l'alcool, facteurs carcinogènes bien connus, peuvent contribuer à la transformation maligne observée dans le LPB mais cette dernière touche surtout des femmes qui ne présentent aucune intoxication éthylo-tabagique. D'autres facteurs intervenant dans la carcinogenèse, comme l'infection candidosique, pourraient être impliquées. Elle est fréquente et récidivante dans le LPB et il a été démontré que le Candida albicans participe à la catalyse de N-nitrosobenzylméthylamine et à une éventuelle transformation maligne [25]. Ce carcinogène, combiné à une synthèse élevée de RNA dans l'épithélium pendant le processus de dégradation-cicatrisation caractéristique de LPB, constitue peut-être un environnement favorable pour le développement d'un clone néoplasique [24]. L'inflammation liée à la candidose buccale pourrait également participer au processus de la transformation maligne.

Néanmoins, quelques auteurs contestent le potentiel de transformation maligne du LPB. Ils mettent en doute le diagnostic de LPB et considèrent que les lésions observées au voisinage du carcinome épidermoïde sont des lésions dysplasiques d'aspect lichénoïde [13]. Krutchkoff et coll. [26] ont procédé à une revue de la littérature sur la transformation maligne du LPB de 1950 à 1977 : ils concluent que seulement 16 cas sur 223 sont suffisamment documentés. Dans leurs conclusions ils avancent trois arguments : (1) les éléments cliniques et histologiques pour confirmer le diagnostic de LPB sont souvent insuffisants, (2) le carcinome épidermoïde se développe bien après l'apparition des lésions du LPB, (3) l'anamnèse comporte des notions insuffisantes sur l'exposition aux carcinogènes, en particulier au tabac [26]. Plus récemment Van der Meij et coll. [56] ont réalisé une autre revue de la littérature sur une période allant 1977 à 1997 avec les mêmes critères que Krutchkoff et coll. [26]. Parmi les 98 cas de transformation maligne du LPB publiés pendant cette période, 33 seulement, soit $34 \%$ de cas publiés, ont été considérés comme suffisamment bien documentés. Avec une approche strictement statistique, ils ont calculé que le pour- centage moyen de transformation maligne de LPB est de $1 \%$ sur une période moyenne de 5 ans, soit un taux annuel de $0,2 \%$, et ont trouvé une prévalence du LPB de $1 \%$ à $2 \%$ dans la population sur une période de 15 ans. A partir de ces données, la prévalence annuelle de transformation maligne pour le LPB est de 2 à 4 cas pour 100.000 habitants. Or, dans certaines régions du monde, cette prévalence correspond à celle du carcinome épidermoïde de la cavité buccale : il faut donc réévaluer la prévalence du LPB et/ou son potentiel de transformation maligne ${ }^{[4]}$. Une étude récente a recherché la perte d'hétérozygotie dans le LPB sur les chromosomes 3p, 9p et $17 p$ car ces altérations qui sont fréquemment retrouvées dans les carcinomes épidermoïdes de la cavité buccale, jouent un rôle dans le processus de transformation maligne. Les résultats ont été comparés avec ceux obtenus dans des lésions réactionnelles (d'origine non précisée par les auteurs) et dysplasiques et dans le carcinome épidermoïde. Dans le LPB, les anomalies génétiques sont moins fréquentes que celles observées dans les autres lésions ce qui permet aux auteurs de conclure que le LPB ne constituerait pas une affection présentant un risque de transformation maligne [60].

\section{CONCLUSION}

Au total, les données de la littérature apparaissent contradictoires; probablement, tout d'abord, parce que le stade évolutif du LPB n'est pas défini avec précision dans la plupart des études. Si l'on considère que la transformation maligne survient au stade de LPB atrophique ou sur un EPL, C'est-à-dire après plus d'une dizaine d'années d'évolution pour le LPB idiopathique -, les études statistiques ne peuvent avoir une réelle valeur que si on précise le stade évolutif pour chaque cas inclus dans la cohorte étudiée, à défaut de connaitre exactement la durée d'évolution du LPB car on ignore bien souvent la date précise de son apparition. Par ailleurs, au cours de l'évolution, les lésions érosives peuvent être observées au cours de deux stades bien différents : lors des poussées de la phase d'état, elles sont secon- 
daires à l'inflammation, tandis qu'au stade de LPB atrophique ou au stade d'EPL, elles sont dues à l'atrophie muqueuse. Or, les premières ne sont jamais le siège de transformation maligne d'où l'intérêt de bien préciser le stade évolutif du LPB. Comme dans la littérature, on parle d'érosions sans préciser le stade évolutif, les résultats présentés sont obligatoirement imprécis. De plus le taux de transformation obtenu est très variable car il dépend de la composition de la cohorte étudiée : il est plus élevé si l'on inclut surtout des cas de LPB au stade atrophique, il est plus faible si

\section{RÉFÉRENCES}

1 - Scully C, Beyli M, Ferreiro MC, Ficarra G, Gill Y, Griffiths M, Holmstrup P, Mutlu S, Porter S, Wray D. Update on oral lichen planus: etiopathogenesis and management. Crit Rev Oral Biol Med. 1998 ; 9 : 86-122.

2 - Axell T, Rundquist L. Oral lichen planus: a demographic study. Community Dent Oral Epidemiol 1987 ; 15 : 52-6.

3 - Andreasen JO. Oral lichen planus: a clinical evaluation of 115 cases. Oral Surg Oral Med Oral Pathol 1968 ; $25: 31-42$.

4 - Abraham SC, Ravich WJ, Anhalt GJ, Yardley JH, Wu TT. Esophageal lichen: planus case report and review of the literature. Am J Surg Pathol 2000 ; 12 : 1678-82.

5 - Ramer MA, Altchek A, Deligdisch L, Phelps R, Montazem A, Buonocore PM. Lichen planus and the vulvovaginal - gingival syndrome. J Periodontol 2003 ; $74: 1385-93$.

6 - Eisen D. The evaluation of cutaneous, genital, scalp, nail, esophageal and ocular involvement in patients with oral lichen planus. Oral Surg Oral Med Oral Pathol Oral Radiol Endod 1999 ; 88 : 431-6.

7 - Cernea P, Kuffer R, Brocheriou C. L'epithélioma sur lichen plan buccal. Act Odont Stoma 1971 ; 96 : 47390.

8 - Samson J, Carrel J-P, Gabioud F. Le lichen plan buccal. Rev Med Suisse Romande 1998 ; 118 : 57-60.

9 - Malherbe E, Drocco C, Lombardi T. Lichen plan buccal et hépatite C. Real Clin 1997 ; 8 : 183-90.

10 - Kuffer R, Lombardi T. Erosion and ulceration occurring on oral lichen planus. Comments on the article "Erosive lichen planus: what is this?" by A. Rebora. Dermatology 2003 ; $207: 340-1$.

11 - Boisnic S, Frances C, Blanchet M-C, Szpirglas H, Charpentier Y. Immunohistochemical study of oral lesions of lichen planus: diagnostic and pathophysiologic aspects. Oral Surg Oral Med Oral Pathol 1990 ; $70: 462-5$.
I'on inclut surtout des cas de LPB à la phase d'état. Enfin les auteurs qui contestent le potentiel de transformation maligne, le font le plus souvent sur la base d'une revue de la littérature et ne font aucune référence à leur expérience clinique personnelle. Et surtout ces auteurs n'apportent pas de réponse aux caractères spécifiques de cette transformation maligne : absence des facteurs carcinogènes classiques, localisations différentes, passage fréquent par le stade de carcinome verruqueux, prédominance de la forme bien différenciée, foyers de transformation multiples.

12 - Lodi G, Scully C, Carrozzo M, Griffiths M, Sugerman P, Thongprasom K. Current controversies in oral lichen planus: report of an international consensus meeting. Part1. Viral infections and etiopathogenesis. Oral Surg Oral Med Oral Pathol Oral Radiol Endod 2005 ; 100 : 40-51.

13 - Mignona MD, Fedele S, Russo L, Muzio L, Bucci E. Immune activation and chronic inflammation as the cause of malignancy in oral lichen planus. Is there any evidence? Oral Oncol 2004 ; 40 : 120-30.

14 - Sugerman PB, Satterwhite K, Bigby M. Autocytotoxic T-cell clones in lichen planus. Br J Dermatol $2000 ; 142$ : 449-56.

15 - McCartan BE, McCreary CE. Oral lichenoid drug eruptions. Oral Dis 1997 ; 3 : 58-63.

16 - Issa Y, Duxbury AJ, Macfarlane TV, Brunton PA. Oral lichenoid lesions related to dental restorative materials. Br Dent J 2005 ; 198 : 361-6.

17 - Bolewska J, Hansen HJ, Holmstrup P, Pindborg JJ, Stangerup M. Oral mucosal lesions related to silver amalgam restorations. Oral Surg Oral Med Oral Pathol $1990 ; 70: 55-8$

18 - Ostman PO, Anneroth G, Skoglund A. Amalgam-associated oral lichenoid lesion reactions. Clinical and histologic changes after removal of amalgam fillings. Oral Surg Oral Med Oral Pathol Oral Radiol Endod 1996 ; $81:$ 459-65.

19 - Lamey PJ, Gibson J, Barclay SC, Miller S. Grinspan's syndrome: a drug-induced phenomenon ? Oral Surg Oral Med Oral Pathol $1990 ; 70$ : 184-5.

20 - Mignona MD, Lo Muzio L, Favia G, Mignona RE, Carbone R, Bucci E. Oral lichen planus and HCV infection: a clinical evaluation of 263 cases. Int J Dermatol $1998 ; 37: 575-8$.

21 - Nagao Y, Sata M, Tanikawa K, Itoh K, Kameyama T. Lichen planus and hepatitis $C$ in nothern Kyushu region of Japan. Eur J Clin Invest 1995 ; 25 : 910-4. médecine

buccale

chirurgie

buccale

VOL. $13, \mathrm{~N}^{\circ} 1$ 2007

page 27 
22 - Ingafou M, Porter SR, Scully C, Teo GC. No evidence of $\mathrm{HCV}$ infection or liver disease in english patients with oral lichen planus. Int J Oral Maxillfac Surg 1998 ; 27 : 65-6.

23 - Campisi G, Giovannelli L, Arico P, Lama A, Di Liberto C, Ammatuna P, D'Angelo M. HPV DNA in clinically different variants of oral leukoplakia and lichen planus. Oral Surg Oral Med Oral Pathol Oral Radiol Endod $2004 ; 98: 705-11$.

24 - Nakamura S, Hiroki A, Shinohara M, Gondo H, Ohyama Y, Mouri T, Sasaki M, Shirasuna K, Harada M, Niho Y. Oral involvement in chronic graft-versus-host disease after allogeneic bone marrow transplantation. Oral Surg Oral Med Oral Pathol Oral Radiol Endod 1996 ; 82 : 556-63.

25 - Sugerman PB, Savage NW. Oral lichen planus: causes, diagnosis and management. Aust Dent J 2002 ; 47 : 290-7.

26 - Lodi G, Scully C, Carrozzo M, Griffiths M, Sugerman $\mathrm{PB}$, Thongprasom K. Current controversies in oral lichen planus: report of an international consensus meeting. Part 2. Clinical management and malignant transfor- médecine buccale chirurgie buccale

VOL. $13, \mathrm{~N}^{\circ} 1$ 2007

page 28 mation. Oral Surg Oral Med Oral Pathol Oral Radiol Endod. $2005 ; 100: 164-78$.

27 - Sugerman PB, Savage NW, Walsh LJ, Zhao ZZ, Zhou XJ, Khan A, Seymour GJ, Bigby M. The pathogenesis of oral lichen planus. Crit Rev Oral Biol Med 2002 ; 13 : 350-65.

28 - Vallejo MJ, Huerta G, Cerero R, Seoane JM. Anxiety and depression as risk factors for oral lichen planus. Dermatology $2001 ; 203$ : 303-7.

29 - Epstein JB, Wan LS, Gorsky M, Zhang L. Oral lichen planus: progress in unterstanding its malignant potential and the implications for clinical management. Oral Surg Oral Med Oral Pathol Oral Radiol Endod 2003 ; $96: 32-7$.

30 - Kaliakatsou F, Hodgson TA, Lewsey JD, Hegarty AM, Porter SR. Management of recalcitrant ulcerative oral lichen planus with topical tacrolimus. J Am Acad Dermatol $2002 ; 46: 35-41$.

31 - Hatchuel DA, Peters E, Lemmer J, Hille JJ, McGaw WT. Candidal infection in oral lichen planus. Oral Surg Oral Med Oral Pathol $1990 ; 70: 172-5$.

32 - Setterfield JF, Black MM, Challacombe SJ. The management of oral lichen planus. Clin Exp Dermatol 2000 ; $25: 176-82$.

33 - Kuffer R. Traitement du lichen plan buccal. I. Généralités, les particularités évolutives du lichen plan buccal, le bilan avant traitement. Rech Dermatol 1988 ; 1 : 496506.

34 - Hallopeau H. Sur un cas de lichen de Wilson gingival avec néoplasie voisine dans la région maxillaire. Bull Soc Fr Dermatol Syphiligr $1910 ; 17: 32$.

35 - Krutchkoff DJ, Cutler L, Lascowski S. Oral lichen planus: the evidence regarding potential malignant transformation. J Oral Pathol $1978 ; 7: 1-7$.
36 - Franck JM, Young AW Jr. Squamous cell carcinoma in situ arising within lichen planus of the vulva. Dermatol Surg 1995 ; 10 : 890-4.

37 - Calabrese C, Fabbri A, Benni M, Areni A, Scialpi C, Miglioli M, Di Febo G. Squamous cell carcinoma arising in esophageal lichen planus. Gastrointest Endosc 2003; $4: 596-9$.

38 - Manz B, Paasch U, Sticherling M. Squamous cell carcinoma as a complication of long-standing hypertrophic lichen planus. Int J Dermatol. 2005 ; 44 : 773-4.

39 - Silverman S Jr, Gorsky M, Lozada-Nur F. A prospective follow-up study of 570 patients with oral lichen planus: persistence, remission, and malignant association. Oral Surg Oral Med Oral Pathol 1985 ; 60 : 30-4.

40 - Holmstrup P, Thorn JJ, Rindum J, Pindborg JJ. Malignant development of lichen planus-affected oral mucosa. J Oral Pathol $1988 ; 17: 219-25$.

41 - Silverman S, Gorsky M, Lozada-Nur F, Giannoti K. A prospective study of findings and management in 214 patients with oral lichen planus. Oral Surg Oral Med Oral Pathol $1991 ; 72:$ :665-70.

42 - Sigurgeirsson B, Lindelöf B. Lichen planus and malignancy. Arch Dermatol 1991; 127 : 1684-8.

43 - Voûte ABE, De Jong WFB, Schulten EAJM, Snow GB, Van der Waal I. Possible premalignant character of oral lichen planus: the Amsterdam experience. J Oral Pathol Med $1992 ; 21$ : 326-9.

44 - Barnard NA, Scully C, Evenson JW, Cunningham S, Porter SR. Oral cancer development in patients with oral lichen planus. J Oral Pathol Med 1993; 22 : 421-4.

45 - Hietanen J, Paasonen MR, Kuhlefelt M, Malmström M. A retrospective study of oral lichen planus patients with concurrent or subsequent development of malignancy. Oral Oncol $1999 ; 35$ : 278-82.

46 - Mignona MD, Lo Muzio L, Lo Russo L, Fedele S, Ruoppo E, Bucci E. Clinical guidelines in early detection of oral squamous cell carcinoma arising in oral lichen planus: a 5-year experience. Oral Oncol 2001 ; 37 : 262-7.

47 - Van der Meij EH, Schepman KP, Van der Waal I. The possible premalignant character of oral lichen planus and lichenoid lesions: a prospective study. Oral Surg Oral Med Oral Pathol Oral Radiol Endod 2003 ; 96 : 164-71.

48 - Bornstein MM, Kalas L, Lemp S, Altermatt HJ, Rees TD, Buser D. Oral lichen planus and malignant transformation: a retrospective follow-up study of clinical and histopathologic data. Quintessence Int 2006 ; 37 : 261 71.

49 - Kaltz RW, Brahim J S, Travis W D. Oral squamous cell carcinoma arising in a patient with long-standing lichen planus. Oral Surg Oral Med Oral Pathol $1990 ; 70$ : 282-5.

50 - Silverman S. Oral lichen planus: a potentially premalignant lesion. J Oral Maxillofac Surg 2000 ; 58 : 12868. 
51 - Pindborg JJ, Reichart PA, Smith CJ, Van der Waal I. Histological typing of cancer and precancer of the oral mucosa (p 29-30). In WHO international histological classification of tumours, 2nd ed, Springer, Berlin, 1997.

52 - Pimenta FJ, Pinheiro MD, Gomez RS. Expression of hMSH2 protein of the human DNA mismatch repair system in oral lichen planus. Int J Med Sci $2004 ; 1$ : 146-51.

53 - Whyte DA, Broton CE, Shillitoe EJ. The unexplained survival of cells in oral cancer: what is the role of p53? J Oral Pathol Med 2002 ; 31 : 125-33.

54 - Valente G, Pagano M, Carrozzo M, Carbone M, Bobba V, Palestro G, Gandolfo S. Sequential immunohistochemical p53 expression in biopsies of oral lichen planus undergoing malignant evolution. J Oral Pathol Med $2001 ; 30: 135-40$.

55 - Acay RR, Felizzola CR, Soares de Araujo N, Machado de Sousa SO. Evaluation of proliferative potential in oral lichen planus and oral lichenoid lesions using immunohistochemical expression of p53 and Ki67. Oral Oncol 2006 ; 42 : 475-80.
56 - Krogh P, Hand B, Holmstrup P. Possible mycological etiology of oral mucosal cancer: catalytic potential of infecting Candida albicans and other yeasts in production of N-nitrosobenzylmethylamine. Carcinogenesis $1987 ; 8$ : 1543-8.

57 - Kaplan B, Barnes L. Oral lichen planus and squamous carcinoma. Case report and update of the literature. Arch Otolaryngol 1985 ; 111 : 543-7.

58 - Eisenberg E. Oral lichen planus: a benign lesion. J Oral Maxillofac Surg $2000 ; 119$ : 1278-85.

59 - Van der Meij EH, Schepman KP, Smeele LE Van der Wal JE, Bezemer PD, Van der Waal I. A review of the recent literature regarding malignant transformation of oral lichen planus. Oral Surg Oral Med Oral Pathol Oral Radiol Endod 1999 ; 88 : 307-10.

60 - Zhang L, Michelsen C, Cheng X, Zeng T, Priddy R, Rosin MP. Molecular analysis of oral lichen planus: a premalignant lesion? Am J Pathol 1997 ; 151 : 323-7. médecine buccale chirurgie buccale

VOL. $13, \mathrm{~N}^{\circ} 1$ 2007

page 29 